\title{
lodination on Tyrosine Residues During Oxidation with Sodium Periodate in Solid Phase Extraction of $\mathbf{N}$-linked Glycopeptides
}

\author{
Alejandro M. Cohen, Ripsik Kostyleva, Kenneth A. Chisholm, Devanand M. Pinto \\ National Research Council, Institute for Marine Biosciences, 1411 Oxford St., Halifax, Nova Scotia, Canada
}

\begin{abstract}
Solid-phase extraction of N-linked glycopeptides (SPEG) using hydrazide-modified supports has become a common sample preparation procedure in glycoproteomic experiments. We demonstrate that iodination of tyrosine residues occur in SPEG as a side reaction during an oxidation step with sodium periodate. MS/MS analysis of oxidized bovine serum albumin and carbonic anhydrase digests revealed a characteristic shift of $m / z 125.9$ on all $\mathbf{y}$ and $\mathbf{b}$ fragment ions containing the modified tyrosine residues. Selected reaction monitoring (SRM) measurements showed that the peak intensity from of the iodinated peptides increased during the course of oxidation. After an hour of oxidation, SRM analysis revealed that the strongest signal from an iodinated peptide was approximately one-tenth of the intensity of the corresponding unmodified peptide. lodinated tyrosine residues were also identified in serum samples subjected to SPEG and analyzed by LC-ESI-MS/MS. We recommend assessing this side reaction by including iodotyrosine as a variable modification when performing database searches on SPEG experiments. For SRM-based acquisitions, we encourage the avoidance of tyrosine-containing glycopeptides or, if this is not practical, monitoring transitions that contain the potential modified iodinated tyrosine residue to monitor the presence of the iodinated form of the glycopeptide.
\end{abstract}

Key words: Glycoproteomics, lodination, N-linked glycoproteins, Hydrazide modified solid phase extraction, Oxidation, Sodium periodate

\section{Introduction}

$\mathrm{T}$ he field of proteomics has evolved into different areas of sub-specialization. Among these, glycoproteomics has attracted much attention due to the critical role glycosylated proteins play in diverse biochemical processes. In addition, glycoproteins have become a common target for biomarker research [1] because glycosylation is a common posttranslational modification (PTM) found in membrane and secreted proteins.

Electronic supplementary material The online version of this article (doi:10.1007/s13361-011-0262-1) contains supplementary material, which is available to authorized users.

Correspondence to: Devanand Pinto; e-mail: dev.pinto@nrc.ca
One of the main challenges in glycoproteomics is the development of sample preparation techniques for enriching glycoproteins and glycopeptides. Presently, two distinct approaches have been adopted for this purpose. Lectin-affinity methods have been used for decades and still offer a convenient way to selectively extract different types of glycan structures [2]. In 2003, an alternative approach using hydrazide modified solid phase supports (SPEG) was proposed [3]. This technique has been adopted by many laboratories, as suggested by the 424 citations received by the original paper at the time this manuscript was prepared. A glyco-proteomic database (http://www.unipep.org) has evolved from the recognition and widespread use of this technique [4]. Furthermore, recent technical developments have increased the throughput of this technique by using superparamagnetic 
silica particles in microtiter plates [5] and automated magnetic particle processors [6].

The sample preparation for SPEG includes a critical oxidation step with sodium periodate. During this reaction, vicinal cis-diol groups in the $\mathrm{N}$-glycan side chains of glycoproteins/peptides are oxidized to aldehydes and then covalently coupled to the hydrazide modified solid support. Following incubation with an endoglycosidase, the N-linked peptides are released with the concomitant conversion of asparagine to aspartic acid residues. Interestingly, this same oxidation step has been used to evaluate a sequence-specific chemistry to capture peptides that contain N-terminal serine or threonine residues [7]. In this procedure, sodium periodate was used to oxidize the 1,2-amino alcohol groups of Nterminal serine and threonine, which then were coupled to biotin hydrazide. The conjugated peptides were finally extracted using streptavidin-coated magnetic beads before analysis by LC-MS/MS.

Dealing with sample complexity is one of the major challenges in proteomics and biomarker research. SPEG has successfully addressed this issue by isolating and enriching the subset of N-linked glycopeptides. Herein, we discuss the formation of iodinated tyrosine residues during the sample preparation of the SPEG procedure. This modification occurs unexpectedly as a side reaction during the oxidation of protein digests with sodium periodate. Although tyrosine does not participate in any known glycosylation motif, it is found in some of the N-linked glycopeptides, which result from the proteolytic digestion of biological samples. Therefore, iodination in these peptides would affect both qualitative and quantitative analysis using SPEG.

\section{Experimental}

\section{Chemicals and Reagents}

Affi-G $\mathrm{Hz}$ beads and Bradford protein assay kit were obtained from Biorad (Mississauga, ON, Canada), PNGaseF from New England Biolabs (Ipswich, MA, USA), and sequencing grade trypsin from Promega (Madison, WI, USA). All other reagents (sodium periodate, tris(2carboxyethyl)phosphine (TCEP), iodoacetamide, potassium phosphate, ammonium bicarbonate, urea, sodium dodecyl sulphate, (SDS), hydrochloric acid, acetic acid, trifluoroacetic acid (TFA), sodium acetate, fetuin, Glu${ }^{1}$-fibrinopeptide B (GluFib), bovine carbonic anhydrase (CA), and bovine serum albumin (BSA) were obtained from Sigma (St. Louis, MO, USA). Serum samples were obtained with consent from volunteers participating in a study approved by the local and NRC research ethics board.

\section{Oxidation of Protein Digests}

The oxidation of BSA and CA was done following the sample preparations steps according to the extraction of Nlinked glycopeptides from Tian et al. [8], with slight modifications. Briefly, $1 \mathrm{mg}$ of BSA was reduced with DTT (5 mM final concentration) for $1 \mathrm{~h}$ at $60{ }^{\circ} \mathrm{C}$ and then alkylated with iodoacetamide (12 $\mathrm{mM}$ final concentration) at room temperature for $30 \mathrm{~min}$ in the dark. CA was neither reduced nor alkylated, since it does not contain any cysteine residues. Both proteins were digested with trypsin at a 1:50 enzyme-to-protein ratio, overnight at $37{ }^{\circ} \mathrm{C}$, with gentle rotation. Samples were desalted by solid phase extraction (SPE) HLB cartridges (\#186000383; Waters, Milford, MA, USA) according to the manufacturer's instructions. The peptides were collected in $1.5 \mathrm{~mL}$ of $50 \%$ acetonitrile in $0.1 \%$ TFA.

A time-course oxidation was performed by adding sodium periodate $(10 \mathrm{mM}$ final concentration in the dark at room temperature) to BSA and CA digests for 15, 30, 45, and $60 \mathrm{~min}$ in separate reaction tubes. An oxidation control (no sodium periodate) was run simultaneously and labeled as time 0 min for plotting purposes. The oxidation at each time point was stopped by a 10 -fold dilution with water and immediately followed by a SPE clean-up step. Samples were finally dried with a SpeedVac $\left(20{ }^{\circ} \mathrm{C}\right)$ and reconstituted in $20 \mu \mathrm{L}$ of a water:acetonitrile:formic acid (95:5:0.1) solution containing $40 \mathrm{fmol} / \mu \mathrm{L} \mathrm{Glu}^{1}$-fibrinopeptide $\mathrm{B}$ used as an internal standard.

\section{Extraction of N-Linked Glycopeptides}

The extraction of N-linked glycopeptides from human serum was performed according to the protocol as described by Tian et al. [8]. Briefly, serum samples were diluted in denaturing buffer containing $8 \mathrm{M}$ urea, $0.4 \mathrm{M}$ ammonium bicarbonate and $0.1 \%$ SDS, reduced with $10 \mathrm{mM}$ TCEP at $60{ }^{\circ} \mathrm{C}$ for $1 \mathrm{~h}$, alkylated in $12 \mathrm{mM}$ iodoacetamide at room temperature for $30 \mathrm{~min}$ in the dark, and finally digested with trypsin overnight at $37^{\circ} \mathrm{C}$. Peptide digests were oxidized with sodium periodate at a final concentration of $10 \mathrm{mM}$ for $1 \mathrm{~h}$ at room temperature in the dark. Following oxidation the samples were coupled to Affi- $\mathrm{G} \mathrm{Hz}$ beads and washed. The $N$-glycosites were released by overnight incubation with $2 \mu \mathrm{L}$ of PNG-ase F at $37^{\circ} \mathrm{C}$ on an orbital tube rotator. Supernatants were collected, washed and dried out on a SpeedVac $\left(20^{\circ} \mathrm{C}\right)$, and finally reconstituted in $20 \mu \mathrm{L}$ of a water:acetonitrile:formic acid (95:5:0.1) solution containing $40 \mathrm{fmol} / \mu \mathrm{L}$ of GluFib.

\section{MS and MS/MS Analysis}

\section{Data-dependent Acquisition}

The oxidized digests from the time course experiments were analyzed by LC-ESI-MS/MS on a Waters Q-TOF Premier (Milford, MA, USA) equipped with a nano-electrospray source and nano-lockspray interface. A lock mass solution of Glu-fibrinopeptide B $(200 \mathrm{fmol} / \mu \mathrm{L})$ was sampled at a frequency of 60:1 (sample-to-reference ratio). A potential of $2800 \mathrm{~V}$ was applied to a $30 \mu \mathrm{m}$ i.d. tapered electrospray tip (FS360-75-30-N-C12; New Objective, Woburn, MA, 
USA). Cone voltage was set to $30 \mathrm{~V}$ and source temperature set to $100{ }^{\circ} \mathrm{C}$.

Chromatographic separations were conducted using a Waters nano-Acquity UPLC system equipped with two $15 \mathrm{~cm} \times 100 \mu \mathrm{m}$ Onyx Monolithic $\mathrm{C}_{18}$ columns in series (Phenomenex, Torrance, CA, USA). The separation was carried out using a linear gradient from $5 \%$ B to $30 \%$ B over 53 min then increasing to $90 \%$ B over 5 min (A: $0.1 \%$ formic acid in water, B: $0.1 \%$ formic acid in acetonitrile) at $1 \mu \mathrm{L} / \mathrm{min}$.

All data acquisition was performed using MassLynx ver. 4.1 (Waters Milford, MA, USA). Data was acquired using data dependent acquisition (DDA) where the $\mathrm{m} / \mathrm{z}$ values of tryptic peptides were measured using a MS scan, followed by collision induced dissociation (CID) MS/MS of the four most intense peaks.

The serum samples analyzed by SPEG were additionally analyzed on an AB/SCIEX QTrap 4000 mass spectrometer coupled to an Agilent 1100 HPLC system equipped with two $15 \mathrm{~cm} \times 100 \mu \mathrm{m}$ Onyx Monolithic $\mathrm{C}_{18}$ columns in series (Phenomenex, Torrance, CA, USA) operated at $35{ }^{\circ} \mathrm{C}$, running an information dependent acquisition method.

\section{Selected Reaction Monitoring Acquisitions}

A selected reaction monitoring method was developed using MRMPilot software (AB SCIEX, Foster City, CA, USA). All BSA and CA tryptic derived peptides containing tyrosine residues were targeted. Transitions containing iodinated and native non-iodinated tyrosine residues were selected for each of these peptides. The details of the acquisition method are shown in Online Resource 1.

Serum digests were analyzed in triplicate injections of $3 \mu \mathrm{L}$ per sample by electrospray LC-MS/MS using an Agilent 1100 HPLC system equipped with two $15 \mathrm{~cm} \times$ $100 \mu \mathrm{m}$ Onyx Monolithic $\mathrm{C}_{18}$ columns in tandem (Phenomenex, Torrance, CA, USA) operated at $35^{\circ} \mathrm{C}$. The separation was carried out using a linear gradient from $2 \% \mathrm{~B}$ to $35 \% \mathrm{~B}$ over 20 min then increasing to $95 \% \mathrm{~B}$ over 5 min at $2 \mu \mathrm{L} /$ min. The HPLC was interfaced to an AB/SCIEX QTrap 4000 mass spectrometer via a nanoflow source. A potential of $5500 \mathrm{~V}$ was applied to the $30 \mu \mathrm{m}$ i.d. tapered electrospray tip (FS360-75-30-N-C12; New Objective, Woburn, MA, USA). The tuning parameters were set to declustering potential (DP) $70 \mathrm{~V}$, entrance potential (EP) $10 \mathrm{~V}$, interface heater temperature (IHT) $150{ }^{\circ} \mathrm{C}$, curtain gas (CUR) $10 \mathrm{psi}$. Data were acquired with Analyst 1.5 software, using a selected reaction monitoring method (SRM), which was developed using MRMPilot ver. 2.0 (AB SCIEX, Foster City, CA, USA). The data acquired with the SRM method was analyzed using MultiQuant ver. 2.0 software $(\mathrm{AB}$ SCIEX, Foster City, CA, USA). For each transition monitored, the relative area ratios were obtained by dividing the peak areas of the target and the corresponding Glufib- $y_{9}$ transition, respectively. The relative area ratios of the transitions originating from the same precursor were added together and averaged for duplicate injections.

\section{Protein and PTM Identification}

Identification of unknown modifications in the oxidized CA and BSA digests analyzed by LC-MS/MS was done using $\mathrm{MOD}^{\mathrm{i}}$, a search engine that uses mass spectrometry data to identify protein modifications [9]. A local database containing the BSA and CA FASTA files was used for sequence matching. An in-depth analysis was done by selecting the MODmap option. MODmap makes use of MOD's unsuccessful identifications to explore unknown modifications from modified regions in MS/MS spectra [10]. Confirmation of the iodinated peptides was performed using the Global Proteome Machine (GPM) search engine using variable modification gaps of 125.9 on tyrosine residues [11]. This search was performed on the complete bovine protein database to confirm the presence of the modified peptides in a sequence-rich context. Identification of proteins from SPEG experiments on human serum was done using Mascot search engine [12] against the human Swissprot database allowing a maximum of one missed cleavage. Carbamidomethyl cysteine (Cys CAM) was introduced as a fixed modification and iodinated tyrosine was set as a variable modification. Peptides and fragment mass tolerances were set at 0.6 and $0.2 \mathrm{~m} / \mathrm{z}$, respectively.

\section{Results and Discussion}

During the validation of candidate glycoprotein cancer biomarkers employing SPEG, we repeatedly observed product ion spectra containing intense fragment information that could not be assigned to any identified protein using automated protein database identification. Manual de novo sequencing of these spectra suggested the presence of an unexpected gap of $288.95 \mathrm{Da}$, compatible with the mass of an iodinated tyrosine residue. We suspect this modification occurred during the oxidation step with sodium periodate, the only reagent containing iodine (other than iodoacetamide used in the alkylation step). To test our hypothesis, we subjected two test proteins, BSA and CA, to identical digestion and oxidation conditions undergone in the SPEG protocol. Carbonic anhydrase was neither reduced nor alkylated after digestion, since it lacks cysteine residues. Consequently, CA digests were only exposed to sodium periodate as a source of iodine, whereas BSA was also exposed to iodoacetamide during the alkylation step. Simultaneously, a set of non-oxidized BSA and CA digests were run as controls.

The protein digests after 60 min of oxidation and the nonoxidized controls were both analyzed by LC-MS/MS, operated in data dependent acquisition (DDA) mode. Product ion peak lists were generated for each run and analyzed using $\mathrm{Mod}^{\mathrm{i}}$, an online bioinformatic tool that uses mass spectrometry data to identify unexpected post-translational modifications in target proteins. To avoid biased results, no expected fixed or variable modifications were added to the query form. In addition, the LC-MS/MS data 
Table 1. List Of Modifications (Delta Mass) Found on Cysteine and Tyrosine Residues for Control and Oxidized BSA and CA Digests, According to MODmap. The Entry for Each Residue Represents the Number of MS/MS Spectra Containing the Predicted Modifications

\begin{tabular}{lccc}
\hline & Delta mass & \multicolumn{2}{c}{ Residues } \\
\cline { 2 - 4 } & & Cysteine & Tyrosine \\
\cline { 2 - 4 } BSA control & 57 & 125 & 1 \\
& 73 & 0 & 0 \\
BSA oxidized & 126 & 0 & 0 \\
& 57 & 15 & 1 \\
CA control & 73 & 7 & 0 \\
CA oxidized & 126 & 0 & 0 \\
\hline
\end{tabular}

was screened using MODmap, an algorithm that explores rare and unknown modifications from modified regions in MS/MS spectra. The MODmap results are shown as a PTM frequency matrix. Table 1 shows a summary of the most relevant modifications obtained from the samples analyzed. Many of these modifications can be explained by the chemical reactions occurring during the sample preparation step, for example, $\mathrm{m} / \mathrm{z}$ shifts of 57 on cysteine residues due to carbamidomethylation by iodoacetamide. Oxidation of carbamidomethylated cysteine residues were also noted by a mass shift of $73(57+16)$ in the oxidized BSA digests (CA lacks cysteine residues). However, an $\mathrm{m} / \mathrm{z}$ shift of 126 was observed on the tyrosine residues of protein digests that had been oxidized, but absent in the control samples. This mass shift is consistent with the addition of an iodine atom and a loss of hydrogen.

In order to further assess the quality of these assignments, the same peak lists for both the control and oxidized digests were submitted to a bovine database search using X!Tandem search engine from the Global Proteome Machine (GPM). A variable modification of +125.9 Da on tyrosine residues was manually added to the list of modified residues. The two oxidized proteins and their controls were all successfully identified as BSA and CA, respectively, with the highest scores in each search. The iodinated tyrosine residues were observed only in the oxidized protein digests (Table 2). In fact, all the candidate tyrosine residues were observed in both their unmodified and iodinated forms, suggesting that iodination occurs partially under these conditions. An example of an iodinated peptide is shown in Figure 1. The doubly charged precursors for both forms of the peptide LGEYGFQNALIVR at $\mathrm{m} / \mathrm{z} 740.70$ and 803.67 differ by exactly half the mass of the proposed modification. The fragments $\mathrm{y}_{10}, \mathrm{y}_{11}$, and $\mathrm{y}_{12}$ all show the characteristic shift of $m / z$ 125.9. The iodinated tyrosine residue did not seem to significantly alter the remaining fragmentation pattern. Furthermore, a few modified peptides were shown to contain di-iodinated tyrosine residues when the database search was repeated introducing a potential modification of +251.8 on tyrosine residues $\left(\mathrm{Y}^{* *}\right.$ in Table 2$)$.

To assess the formation of the iodinated peptides during the oxidation with sodium periodate, BSA and CA digests

Table 2. List of Iodinated Peptides Found in the Oxidized Digests of BSA and CA Using the GPM Search Engine

\begin{tabular}{|c|c|c|c|}
\hline Sample & Peptide sequence & Exact mass $[\mathrm{M}+\mathrm{H}]^{+}$ & Mass error $(\mathrm{Da})$ \\
\hline BSA & GLVLIAFSQY*LQQC*PFDEHVK & 2618.164 & 0.077 \\
\hline \multirow[t]{12}{*}{ Oxidized } & GLVLIAFSQY"LQQC ${ }^{* \wedge}$ PFDEHVK & 2650.154 & 0.063 \\
\hline & HPYFYAPELLY*YANK & 2014.827 & 0.064 \\
\hline & HPYFY $^{*}$ APELLYY & 1701.652 & 0.057 \\
\hline & HPYFY* APELLY & 1538.589 & 0.051 \\
\hline & FYAPELLY ${ }^{*}$ YANK & 1617.652 & 0.042 \\
\hline & DAFLGSFLYEY*SR & 1693.643 & 0.069 \\
\hline & DAFLGSFLY ${ }^{*}$ EYSR & 1693.643 & 0.051 \\
\hline & RHPEY* AVSVLLR & 1565.712 & 0.045 \\
\hline & LGEY* GFQNALIVR & 1605.695 & 0.026 \\
\hline & MPC ${ }^{* \wedge \wedge}$ TEDY ${ }^{*}$ LSLILNR & 1882.724 & 0.047 \\
\hline & $\mathbf{M}^{*} \mathrm{PC}^{* \wedge} \mathrm{TEDY}^{* * *}$ LSLILNR & 2008.620 & 0.065 \\
\hline & 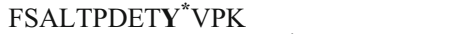 & 1593.637 & 0.055 \\
\hline $\mathrm{CA}$ & AVVQDPALKPLALVY*GEATSR & 2324.118 & -0.021 \\
\hline \multirow[t]{8}{*}{ Oxidized } & AVVQDPALKPLALVY ${ }^{* * *}$ GEATSR & 2450.018 & -0.041 \\
\hline & ALVY $^{*}$ GEATSR & 1192.453 & -0.015 \\
\hline & $\mathbf{M}^{*}$ VNNGHSFNVEY* DDSQDK & 2240.772 & -0.054 \\
\hline & DGPLTGTY*R $^{*}$ & 1105.384 & -0.018 \\
\hline & $\mathbf{Y}^{*}$ AAELHLVHW"NTK & 1723.712 & -0.037 \\
\hline & $\mathbf{Y}^{*}$ GDFGTAAQQPDGLAVVGVFLK & 2379.055 & -0.031 \\
\hline & $\mathbf{Y}^{* *}$ GDFGTAAQQPDGLAVVGVFLK & 2504.955 & -0.041 \\
\hline & $\mathbf{Y}^{*}$ GDFGTAAQQPDGLAVVGVF & 2137.876 & -0.023 \\
\hline
\end{tabular}

$\mathrm{Y}^{*}$ Iodinated tyrosine

$\mathrm{Y}^{* *}$ Di-iodinated tyrosine

$\mathrm{C}^{*}$ Carbamidomethyl cysteine

$\mathrm{C}^{* \wedge}$ Oxidized carbamidomethyl cysteine

$\mathrm{C}^{* \wedge}$ Di-oxidized carbamidomethyl cysteine

$\mathrm{M}^{*}$ Oxidized methionine 


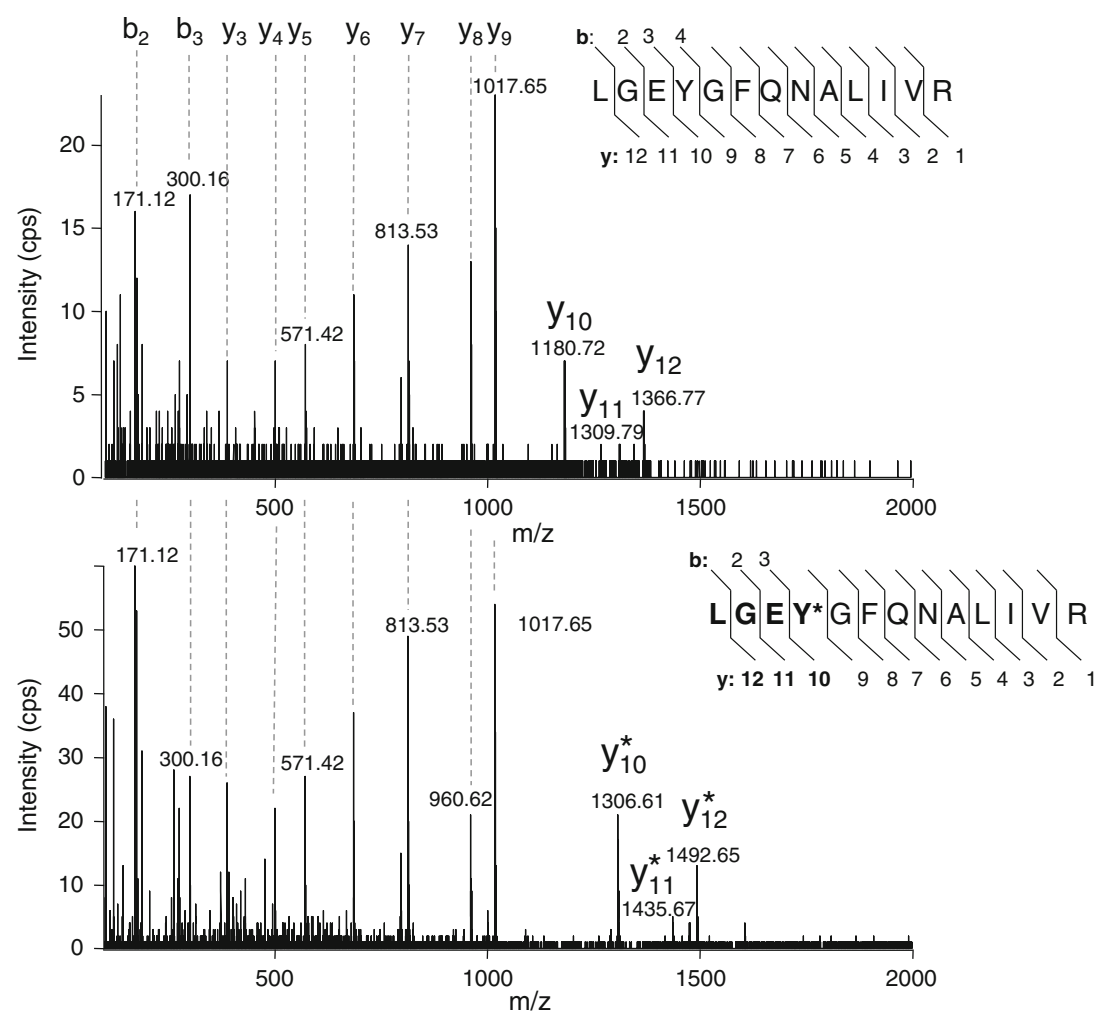

Figure 1. Fragmentation patterns from a control (Top: MS/MS of $[\mathrm{M}+2 \mathrm{H}]^{2+}$ at $\mathrm{m} / \mathrm{z} 740.70$ ) and an oxidized BSA peptide (Bottom: MS/MS of $[\mathrm{M}+2 \mathrm{H}]^{2+}$ at $m / z$ 803.67). lodinated tyrosine residues are labeled as $\mathrm{Y}^{*}$

were subjected to a 0 (no-oxidation control), $15,30,45$, and 60 min oxidation. These samples were analyzed using an SRM method targeting the peptides containing tyrosine residues and their iodinated derivatives, exemplified in Figure 2. Assuming no other reaction is involved, the extent of iodination can be estimated from the decrease in the intensity of the unoxidized form of the peptide. Alternatively, the ionization efficiencies of the iodinated forms could have been measured using synthetic iodinated peptides, unfortunately, to our knowledge, these are not commercially available. The degree of iodination varied among peptides; the signal intensity of unmodified peptide LGEYGFQNALIVR in Figure 2 decreased by $30 \%$ after $60 \mathrm{~min}$ of oxidation, whereas the intensity of another peptide, YICDNQDTISSK, decreased by $50 \%$ (Figure 3). The signal intensities from the XICs of the iodinated peptides were approximately two orders of magnitude weaker than the non-iodinated forms, (Figure 3). Iodination was also observed on peptides containing more than one tyrosine residues. For example, after $60 \mathrm{~min}$ of oxidation, the peptide DAFLGSFLYEYSR was observed in both of its iodinated forms, i.e., containing mono iodo tyrosine in either position. Interestingly, both iodinated forms of this peptide could be distinguished by a small difference in their retention times, as shown by the diagnostic transitions (Figure 4). A SRM method was also developed to monitor the transitions of this peptide iodinated simultaneously at both positions; however, no clear signal was obtained (data not shown).

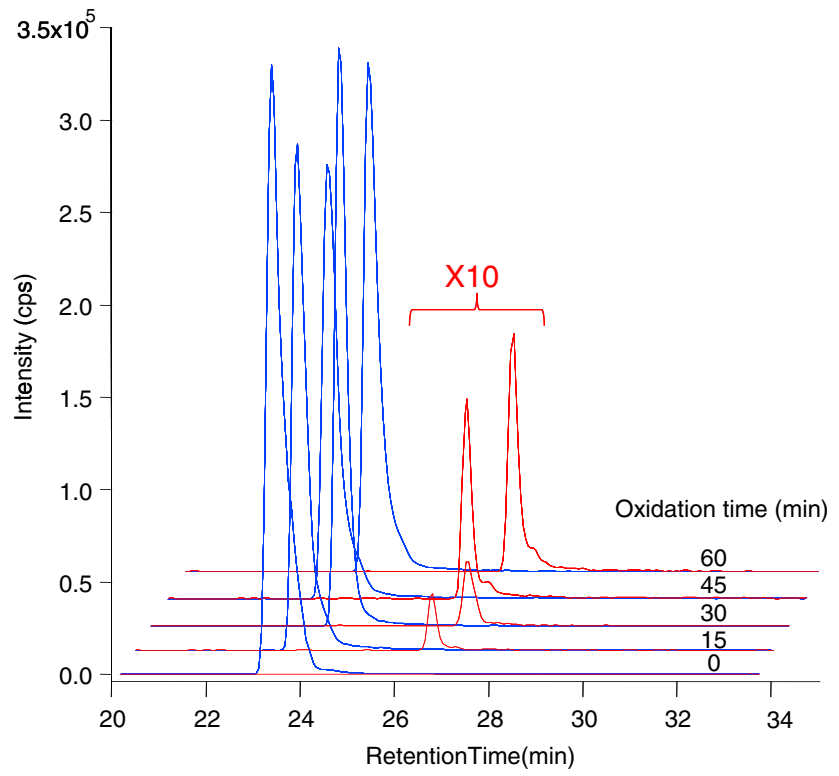

Figure 2. Formation of LGEYGFQNALIVR and LGEY*GFQNALIVR peptides during the oxidation-time course experiment. The SRM-extracted ion chromatograms of the $[\mathrm{M}+2 \mathrm{H}]^{2+} \rightarrow \mathrm{y}_{9}$ transition is shown for both the unmodified peptide (blue trace) and the iodinated $\left(\mathrm{Y}^{*}\right)$ peptide (red traces). Note that the traces are offset vertically and horizontally $(0.5 \mathrm{~min})$ and the signal of the iodotyrosine peptides was multiplied by 10 to improve visualization 

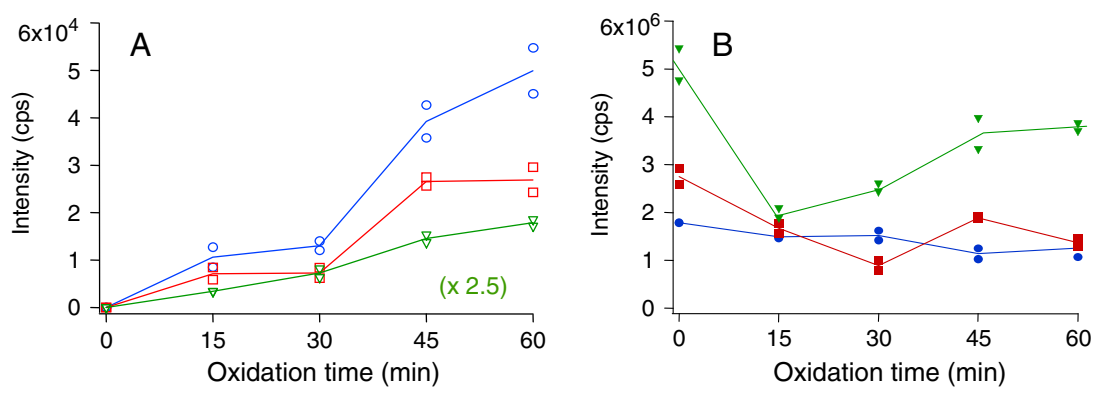

Figure 3. Levels of BSA peptides LGEYGFQNALIVR (blue circles), YIC[CAM]DNQDTISSK (red squares) and CA peptide DGPLTGTYR (green triangles) during the oxidation time-course experiment. The SRM signals are shown for both the (a) iodinated peptides and (b) and unmodified peptides. The time point equal to 0 min corresponds to the non-oxidized control. The intensity of the iodinated form of the CA peptide was multiplied by 2.5 for clarity

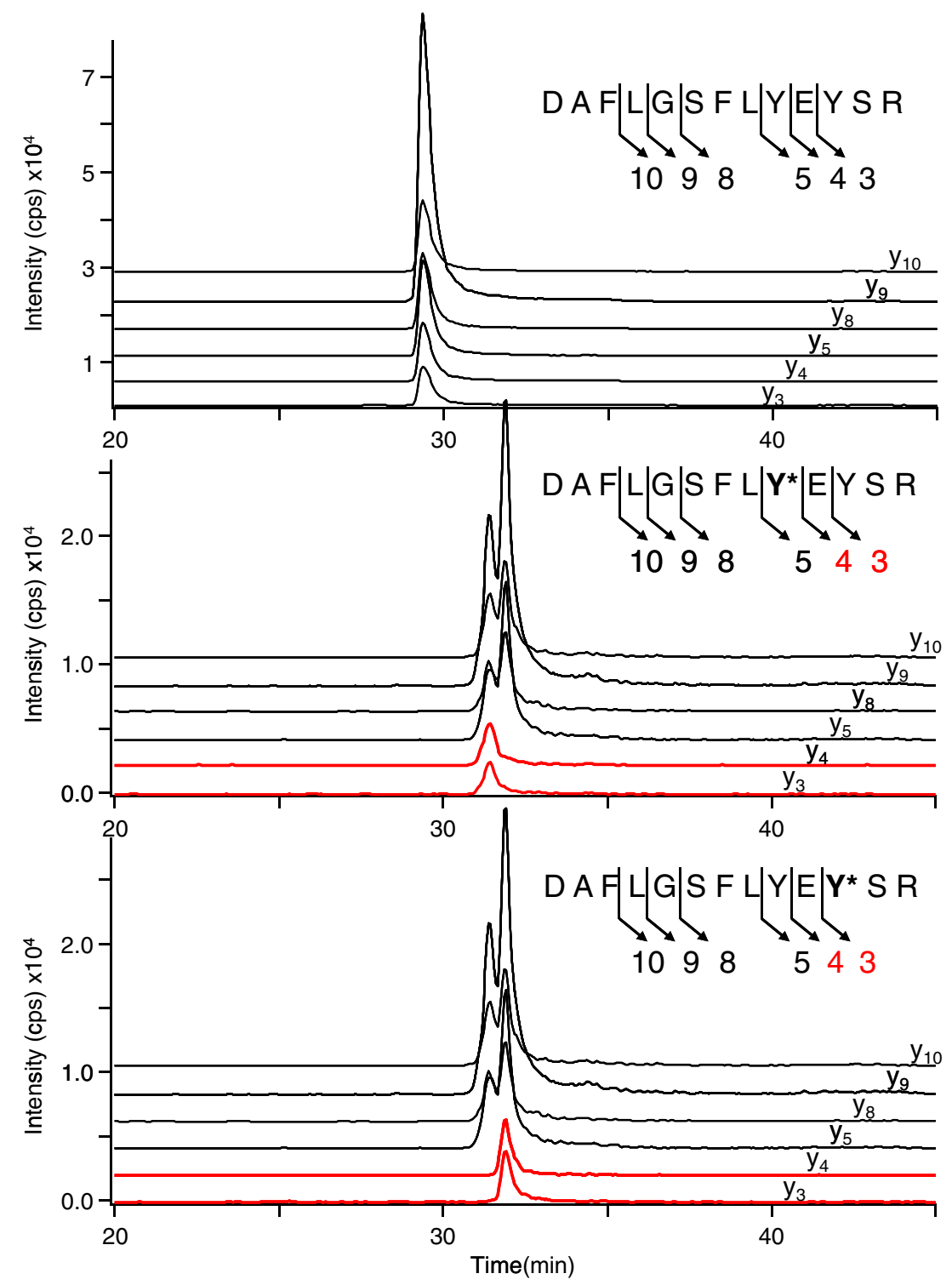

Figure 4. SRM acquisitions of peptide DAFLGSFLYEYSR after $60 \mathrm{~min}$ of oxidation. The top chromatogram shows the XICs of the non-iodinated peptide $(\mathrm{Rt}=29.5 \mathrm{~min})$. The middle and bottom chromatograms show the XICs for peptides iodinated in positions 5 and 3 , respectively, (Rt=31.4 and $31.9 \mathrm{~min}$ ). In red, the traces corresponding to the transitions of the distinctive of $\mathrm{y}_{3}$ and $y_{4}$ fragment ions. Note that the traces from transitions monitoring fragments $y_{5}$ to $y_{10}$ are indistinguishable in middle and bottom chromatograms 
Table 3. Mascot Search Results of Iodinated N-Glycosites Extracted from Serum Samples by SPEG, and Analyzed by LC-MS/MS Using a Cut-off Peptide Score of 25

\begin{tabular}{|c|c|c|c|c|}
\hline Protein & Acc \# & Peptide & Mass diff. & Score \\
\hline$\alpha-1$-Acid glycoprotein & P02763 & QDQC*IY*N*TTYLNVQR & 0.28 & 63 \\
\hline$\alpha-1$-Antitrypsin & P01009 & Y*LGN*ATAIFFLPDEGK & 0.30 & 35 \\
\hline Haptoglobin & P00738 & VVLHPN*Y $*$ SQVDIGLIK & 0.32 & 33 \\
\hline Clusterin & P10909 & LAN*LTQGEDQYY*LR & 0.55 & 68 \\
\hline Ig $\gamma$-1 chain $C$ & P01857 & EEQY*N*STYR & 0.12 & 43 \\
\hline Ig $\gamma-3$ chain $C$ & P01860 & EEQY* $\overline{N * S T F R}$ & 0.31 & 27 \\
\hline
\end{tabular}

$\mathrm{Y}^{*}$ Iodinated tyrosine

$\mathrm{M}^{*}$ Oxidized methionine

$\mathrm{N}^{*}$ Deamidated asparagine (aspartic acid)

$\mathrm{N} *$ xT/S Consensus motif for $N$-glycosylation

Finally, to test whether the iodination reaction occurs in complex samples we analyzed serum digests subjected to SPEG and acquired by LC-MS/MS. The database searches were repeated using Mascot with iodinated tyrosine in the list of variable modifications. Six iodinated glycoproteins were identified from a total of 68 glycoproteins (Table 3). It should be noted that only a fraction of the N-linked glycosites are expected to contain tyrosine residues, determined by the natural distribution of this amino acid within the trypsin cleavage sites of the proteins. To determine whether this modification was an artifact particular to our experimental setup, we analyzed an external dataset deposited in the Tranche-Proteome Commons repository (https://www.proteomecomons.org, upload by Clauser, Dec. 21, 2009, Broad Institute). This study optimized the performance of glycopeptide capture in human plasma using a similar SPEG procedure and analyzed the extracts on a LTQ-FT mass spectrometer [6]. A database search of this dataset including iodotyrosine as a potential modification identified 27 peptides containing both the signature N-x-S/T glycosylation motif and iodinated tyrosine residues (Online Resource 2). The identification of these peptides in both studies suggests that the proposed modification is common but does not significantly affect the behavior of the iodinated peptides during the downstream steps of SPEG. Furthermore, iodination of the peptides does not seem to interfere with either the ESI process or the CID fragmentation.

The mechanisms of tyrosine iodination have been described in the literature $[13,14]$. Iodination of unsubstituted aromatic rings is an electrophilic aromatic substitution reaction that proceeds spontaneously in the presence of a catalyst. The reaction on phenolic rings is further enhanced by the presence of the $-\mathrm{OH}$ group and does not require a catalyst. The mechanism for iodination occurs through the formation of electrophilic iodine $\left(\mathrm{I}^{+}\right)$, usually obtained by the addition of an oxidizing agent such as nitric acid to the reaction. The periodate oxidation tested in our experiments occurs with the concomitant reduction of the periodate anion; however, the end product of this hemi-reaction in these particular oxidation reactions has not been studied. Nevertheless, the finding of iodinated tyrosine residues suggests that the reduction of the periodate anion might result in the formation of reduced forms of the periodate anion such as $\mathrm{I}^{+}$and $\mathrm{I}_{2}$ and $\mathrm{I}^{-}$, responsible for the iodination reaction. This reaction might be enhanced by trace amounts of reducing agents typically added in proteomics experiments for the reduction of disulphide bonds prior to the digestion of proteins.

\section{Conclusion}

We have observed iodination of peptides containing tyrosine residues in protein digests, which have been subjected to oxidation with sodium periodate. The iodination of oxidized CA digests suggests that sodium periodate is most probably the source of the iodine reactive species. The data obtained from the SRM acquisitions suggest that the signals from iodinated peptides can be as high as $10 \%$ of the intensity compared with that of the corresponding unmodified peptide. However, the precise concentration of these modified peptides cannot be confirmed in the absence of an iodinated standard, which would account for variations in ionization and fragmentation efficiencies. Regardless, these modified peptides are present in sufficient quantity to be detected in DDA experiments of serum samples processed by SPEG.

In summary, we recommend the following guidelines to improve the results of 'bottom-up' proteomics studies in which use sodium periodate is used in the sample preparation: for discovery/qualitative glycoproteomics, iodo-tyrosine should be added to the list of variable modifications in database searching to confirm the presence of the iodinated peptides. In some cases, this may improve protein identification and sequence coverage. For quantitative glycoproteomics using SRM, we discourage the selection of peptides containing tyrosine residues. In the cases where no alternative peptides exist, we suggest monitoring the transitions containing potential iodinated tyrosine residues to assess the extent of the iodination reaction.

\section{Acknowledgments}

The authors acknowledge funding of this work through the Genomics and Health Initiative of the National Research Council (Canada). This is NRC Publication number NRC-7828. 


\section{References}

1. Boland, M., Rudd, P.M.: Disease related glycosylation changes and biomarker discovery: Challenges and possibilities in an emerging field. Editorial. Dis Markers 25(4/5), 189-192 (2008)

2. Hirabayashi, J., Hashidate, T., Kasai, K.: Glyco-catch method: A lectin affinity technique for glycoproteomics. J. Biomol. Tech. 13(4), 205-218 (2002)

3. Zhang, H., Li, X.J., Martin, D.B., Aebersold, R.: Identification and quantification of n-linked glycoproteins using hydrazide chemistry, stable isotope labeling and mass spectrometry. Nat. Biotechnol. 21(6), 660-666 (2003)

4. Zhang, H., Loriaux, P., Eng, J., Campbell, D., Keller, A., Moss, P., Bonneau, R., Zhang, N., Zhou, Y., Wollscheid, B., Cooke, K., Yi, E.C., Lee, H., Peskind, E.R., Zhang, J., Smith, R.D., Aebersold, R.: Unipepa database for human n-linked glycosites: A resource for biomarker discovery. Genome Biol. 7(8), R73 (2006)

5. Zou, Z., Ibisate, M., Zhou, Y., Aebersold, R., Xia, Y., Zhang, H.: Synthesis and evaluation of superparamagnetic silica particles for extraction of glycopeptides in the microtiter plate format. Anal. Chem. 80(4), 1228-1234 (2008)

6. Berven, F.S., Ahmad, R., Clauser, K.R., Carr, S.A.: Optimizing performance of glycopeptide capture for plasma proteomics. $J$. Proteome Res. 9(4), 1706-1715 (2010)
7. Chelius, D., Shaler, T.A.: Capture of peptides with N-terminal serine and threonine: A sequence-specific chemical method for peptide mixture simplification. Bioconj. Chem. 14(1), 205-211 (2003)

8. Tian, Y., Zhou, Y., Elliott, S., Aebersold, R., Zhang, H.: Solid-phase extraction of n-linked glycopeptides. Nat. Protoc. 2(2), 334-339 (2007)

9. Kim, S., Na, S., Sim, J.W., Park, H., Jeong, J., Kim, H., Seo, Y., Seo, J., Lee, K.J., Paek, E.: Modi: A powerful and convenient web server for identifying multiple post-translational peptide modifications from tandem mass spectra. Nucleic Acids Res. 34, W258-W263 (2006). Web Server issue

10. Na, S., Paek, E.: Prediction of novel modifications by unrestrictive search of tandem mass spectra. J. Proteome Res. 8(10), 4418-4427 (2009)

11. Craig, R., Beavis, R.C.: Tandem: Matching proteins with tandem mass spectra. Bioinformatics 20(9), 1466-1467 (2004)

12. Perkins, D.N., Pappin, D.J., Creasy, D.M., Cottrell, J.S.: Probabilitybased protein identification by searching sequence databases using mass spectrometry data. Electrophoresis 20(18), 3551-3567 (1999)

13. Dunford, H.B., Ralston, I.M.: On the mechanism of iodination of tyrosine. Biochem. Biophys. Res. Commun. 116(2), 639-643 (1983)

14. Dunford, H.B., Adeniran, A.J.: The mechanism of the nonenzymatic iodination of tyrosine by molecular iodine. Biochem. Cell Biol. 66(9), 967-978 (1988) 